Amaral Rebouças, A. e107 Amaral Rebouças Moreira, M. e107

Anders, H.-J. e63

Araki, T. el17

Barisoni, L. e92

Barstead, R. e9

Beck, K.-F. e23

Bertram, J.F. e18

Bogaert, T. e9

Branden, M. e9

Braun, M.C. e92

Brodsky, S.V. e46

Buechner, M. e9

Burrow, C. e9

Chaponnier, C. e158

Damario Gomes, M. e107

Dashwood, M.R. e80

Dewulf, N. e9

Doi, S.Q. e134

Donovan, K.L. e125

Eberhardt, W. e23
Eitner, F. e158

Floege, J. e158

Gabbiani, G. e158

Geng, L. e9

Goligorsky, M.S. e46

Goto, H. e27

Grabensee, B. e72

Hammerman, M.R. e58

Han, J.S. e36

Hayashi, M. e117

Heering, P. e72

Heise, G. e72

Hendry, B.M. e129

Hirata, M.H. e134

Hirata, R.D.C. e134

Hirszel, P. e134

Huwiler, A. e23

Hyink, D. e9

Imai, H. e27

Janssen, U. e158

Jeon, U.S. e36

Joo, K.W. e36
Josifovska, T. e141

Kaletta, T. e9

Kashgarian, M. e46

Kerjaschki, D. e158

Kim, G.-H. e36

Kim, J. e36

Kim, Y.S. e36

King, K.V. e9

Knepper, M.A. e36

Kobayashi, R. e27

Komatsuda, A. e27

Kopp, J.B. e92

Kunter, U. e158

Lakkis, M. e3

Lee, J.S. e36

Li, H.-P. e9

Liapis, H. e87

Lu, H. e92

Luckow, B. e63

Machado, M.O. e134

Machida, K. e141

Mack, M. e63
Madej, A. e150

Martinez, G. e18

Meyer-Kirchrath, J. e72

Mobaraki, H. e92

Morishima, N. e117

Na, K.Y. e36

Nakanishi, K. e117

Nauman, A. e150

Nauman, J. e150

Newby, L.J. e80

Nielsen, S. e36

Nonoguchi, H. e141

Ohtani, H. e27

Ong, A.C.M. e80

Ostendorf, T. e158

Owens, J. e92

Pérez de Lema, G. e63

Pfeilschifter, J. e23

Poulsom, R. e53

Puzianowska-Kuznicka, M. e150

Saruta, T. e117
Sawada, K. e27

Schlöndorff, D. e63

Schrör, K. e72

Sellitti, D.F. e134

Sharpe, C.C. e129

Shrivastav, S. e92

Smith, M. e46

Tanski, Z. e150

Tomita, K. e141

Topley, N. e125

Van der Craen, M. e9

Van Geel, A. e9

Vasconcelos Cancherini, D. e107

Vielhauer, V. e63

Wakui, H. e27

Waldner, C. e72

Wilson, P. e9

Wolf, G. e158

Yo, Y. e92

Zhou, J. e3

\title{
Subject Index Vol. 93, 2003
}

\section{Actin e158}

$\alpha$-Actinin-4 e27

Acute vascular rejection e58

Adherens junction e3

Angiogenesis e46

Angiogenin e158

Antibody e63

Apoptosis e92

Aquaporin-2 e36

Autosomal-dominant polycystic disease e 80

- $\quad$ - kidney disease e3

Basolateral redistribution e36

Caenorhabditis elegans e9

Caspase e117

CCR2 e63

CCR5 e63

Celecoxib e72

Cell proliferation e92

- survival genes e87

Cellular transplant e58

Chemo-/mechanosensors e9

Cholesterol metabolism e134

Chronic renal failure e141

Congenital development e87

Co-stimulatory blockade e 58
Cyclooxygenase-2 e72

Cyst formation e3

Cytoskeletal organization e3

Defensins e125

Diabetes mellitus e46

DNA binding e150

Endothelial cell e158

Endothelin e80

Expression e150

FACS 63

Fibrosis e129

Focal adhesions e9

Gene expression e23

- therapy e129

Genetic disorder e3

Genomic analysis e9

Glomerular filtration barrier e27

Glomerulonephritis e158

Glomerulosclerosis e134

Growth hormone e134

GTPase e129

Human renal clear cell cancer e150

3-Hydroxy-3-methylglutaryl coenyzme A reductase e134

Hyperacute rejection e58

Hypoxia e107
Inflammation e23

$\alpha_{\mathrm{v}}$ Integrins e72

Ischemic preconditioning e107

Kidney e72

- disease e134

- explants e46

Low-density lipoprotein receptor e134

Mesangial cells e23

Metabolic acidosis e141

Metanephros el17

Microdissection e141

Mucosal immunity e125

Mutations e9

Nephrogenesis e117

Nephrotoxic serum e92

Neuronal processes e9

Nitric oxide e23

Oxidative stress e107

Oxytocin e36

Parathyroid hormone receptor e141

Passive Heymann nephritis e72

Paxillin e9

Plasticity e53

Podocyte cytoskeleton e27

Polycystic kidney disease e9
Polycystin e3

Protein expression e9

Puromycin aminonucleoside e27

Ras e129

Receptors e80

Redistribution e36

Redox signaling e23

Regeneration e53

Renal disease e 129

- failure e107

- morphogenesis e9

Repair e53

Reverse-transcription competitive polymerase chain reaction e141

Reverse transcription-polymerase chain reaction e134

Rho e129

Signaling e3

- molecules e87

Subtractive hybridization e107

Superoxide e23

Tubulogenesis e3

Urinary tract infection e125

Vasopressin-2 receptor e36

Vitamin D e150

- - receptor e150

\section{KARGER}

(C) 2003 S. Karger AG, Basel

Fax +41613061234

E-Mail karger@karger.ch

www.karger.com 